\title{
The convergence of systemic threads shaping a future South African healthcare dispensation: A technology management perspective
}

\author{
Author: \\ Richard Weeks ${ }^{1}$ \\ Affiliation: \\ ${ }^{1}$ Graduate School of \\ Technology Management, \\ University of Pretoria, \\ South Africa \\ Correspondence to: \\ Richard Weeks \\ Email: \\ richard.weeks@up.ac.za \\ Postal address: \\ PO Box 70563, The Willows \\ 0041, South Africa \\ Dates: \\ Received: 26 Feb. 2013 \\ Accepted: 29 May 2013 \\ Published: 02 Aug. 2013 \\ How to cite this article: \\ Weeks, R., 2013, 'The \\ convergence of systemic \\ threads shaping a future \\ South African healthcare \\ dispensation: A technology \\ management perspective', \\ Acta Commercii 13(1), \\ Art. \#181, 8 pages. \\ http://dx.doi.org/10.4102/ \\ ac.v13i1.181

\section{Copyright:} \\ (C) 2013. The Authors. \\ Licensee: AOSIS \\ OpenJournals. This work \\ is licensed under the \\ Creative Commons \\ Attribution License.
}

Read online:
Orientation: Underpinning healthcare service delivery are a number of support systems. This paper focuses on the development of a healthcare services framework that reflects the systems that need to be integrated, from a technology healthcare support perspective.

Research purpose: The purpose of this paper is gain an understanding of some of the intricacies associated with the management of the transition to a future South African healthcare dispensation, with reference to the convergence of technology, financial healthcare and sociopolitical systems.

Motivation for the study: South Africa is in the process of implementing the National Health Insurance initiative and the approach adopted will have a significant impact on the business model design.

Research design, approach and method: A multidisciplinary literature study was undertaken. In addition, a limited narrative enquiry was also conducted. Practitioners interviewed were from the healthcare, informatics and management and technology sectors respectively. The research study constituted an insight study - analytically descriptive and not statistical in nature.

Main findings: The literature reflects two very contrasting and different business models of healthcare service provision, namely a primarily curative and preventative stance. Each assumes a very different convergence of technology, healthcare, financial and social systems and consequently gives rise to contrasting business models. The dominant model appears to be based on primary healthcare, with a different technology support infrastructure to the previously-adopted curative approach. It is a model that would also appear to necessitate a complex adaptive management approach, necessitating a bottom-up as opposed to a top-down hierarchal management orientation.

Contribution/Value-add: The National Healthcare Insurance initiative entails a very fundamental restructuring of the healthcare infrastructure. The insights gained from this research study could serve as a source of information and reference for South African institutions that will need to implement such systems in the future.

\section{Introduction}

When people think of health, they tend to think narrowly about treatment and care delivered by a healthcare system rather than broadly about a health system that includes policies, products and services aimed at disease prevention and well-being. (World Economic Forum 2013:5)

Reflected in the introductory statement by the World Economic Forum (2013:5) are contrasting points of view regarding healthcare service delivery. The former, more traditional, curative view tends to dominant many a nation's healthcare system, whilst the latter is increasingly deemed to be more sustainable within our current global financially-constrained economy. Much of the current debate on the future of healthcare, according to the World Economic Forum (2013:5), is 'characterized by short-term and siloed thinking and entrenched positions', which discourages conversations about more fundamental changes. A wider discourse has apparently underpinned the South African government's National Health Insurance (NHI) policy document (Department of Health [DoH] 2011a:5), as envisioned therein is a complete transformation of healthcare service provision and delivery, with an accent placed on the need for a comprehensive package of care underpinned by primary healthcare (PHC). The PHC approach to the restructuring of the country's healthcare infrastructure is underscored by the definition attributed to 'health' by the World Health Organization (WHO) (DoH 2011a:23), namely:

Health is not just the absence of disease or infirmity but a state of complete physical, mental and social wellbeing. It is a fundamental human right and the attainment of the highest possible level of health is the most important worldwide social goal whose realisation requires action from many other social and economic sectors in addition to the health sector. 
Translating healthcare into practice within the context of this definition necessitates providing a package of services that extends beyond the curative services traditionally provided by healthcare facilities, cases in point being home-based and school health services. A central facet of these services will be the promotion of healthy lifestyles and prevention, whilst not excluding curative services. This wider orientation attributed to healthcare brings a number of diverse systems within the healthcare management framework together to give rise to a new healthcare dispensation. Systems typical of this approach that previously did not assume such pertinence are, inter alia, that of community-based social systems, healthcare information systems, eHealth and, more pertinently, mobile technologies, to name but a few. A diagrammatic presentation of the various systems constituting a typical healthcare management framework is depicted in Figure 1. It is argued in this paper that these systems are increasingly converging and managing the various interdependencies and interaction taking place between these systems is becoming extremely complex.

Feachem (2000:715), it would seem, concurs with regard to the complexity associated with healthcare service systems management, in stating that ' $h]$ ealth systems policy issues are intrinsically complex'. The researcher further claims that no country has discovered an ideal model for managing these systems (Feachem 2000:715). Murray and Frenk (2000:717), similarly, claim that health systems vary widely in performance and even countries with similar levels of income, education and health expenditure differ in their ability to attain key health goals. The first step in developing a framework to advance the understanding of health system performance, according to Murray and Frenk (2000:717), is to define the boundaries of the health system based on the concept of health action. Health action is defined by the researchers as being any set of activities whose primary intent is to improve or maintain health (Murray \& Frenk 2000:717). Within the context of the South African NHI initiative, the strengthening of the health system will, as alluded to previously, be based in essence on a PHC approach (DoH 2011a:23).

In the ensuing discourse, the focus will be on gaining an understanding of the intricacies associated with the management of the transition to a future PHC-orientated South African healthcare dispensation, with reference to the convergence of technology, as well as the human, legal, financial and socio-political support systems which underpin the healthcare value chain. A multidisciplinary literature review and a restricted narrative enquiry serve as the methodology basis for the research study. The accent in conducting the literature review was on obtaining the most contemporary literature, making use of the IEEE (Institute of Electrical and Electronics Engineers technical database), South African e-publications, medical journals and EbscoHost search platforms. Practitioners interviewed were from the healthcare, informatics, and management and technology sectors respectively. Five healthcare, two management, and four information and communication technology practitioners involved in the design, implementation and

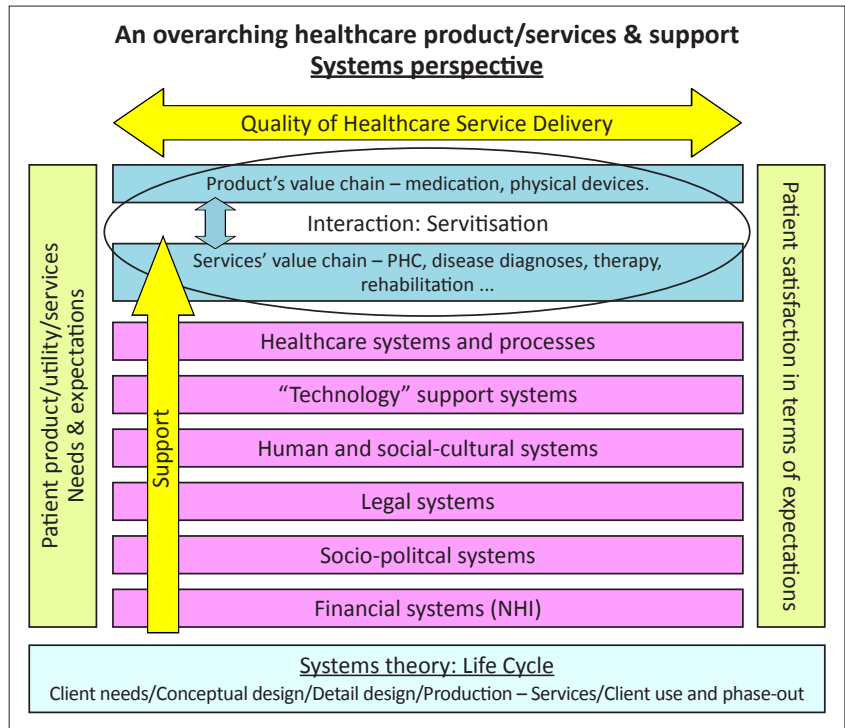

Source: Adapted from Weeks \& Benade 2011

FIGURE 1: A healthcare systems management framework.

use of eHealth solutions at a district hospital and clinic were interviewed during the course of the research study. The interviews were semi-structured and open-ended in order to allow respondents to reflect on aspects that they considered relevant, based on their own extensive experience. In order to learn from their hands on experience it was agreed that the institutions and practitioners concerned would not be identified as in some instances the information could be deemed to be of a sensitive nature.

\section{The transition to a National Health Insurance dispensation: Technology considerations}

The transition to an NHI dispensation has far-reaching consequences for all South Africans. It enables a re-evaluation of the structure and boundaries of existing healthcare systems, as well as how they converge to engender a healthcare dispensation that provides all South Africans with 'access to affordable quality healthcare services regardless of their socio-economic status' (DoH 2011a:4). Acknowledged in the NHI policy document (DoH 2011a:5) is the need for a total transformation of the entire healthcare system. Central to this transformation is the realisation that healthcare has a very fundamental impact on the South African economy and its competitiveness within the global marketplace.

The World Economic Forum's (2012:324) Global Competitiveness Report for 2012-2013 reveals that South Africa's ranking has dropped from 50th to 52nd position in the global ranking index and that poor public health is cited as being one of the problematic factors for conducting business in the country. South Africa's ranking in terms of the fourth pillar relating to health and primary education is of particular concern in terms of the prevalence of malaria, tuberculosis and HIV. Also of note is its poor ranking in relation to primary education - a ranking of 132 out of 144 nations (World Economic Forum 2012:325). The picture that 
emerges in relation to higher education and training is also daunting in that the quality of the education system is ranked 140th globally. An inadequately-educated workforce is in fact cited as being the most problematic factor for conducting business in South Africa (World Economic Forum 2012:324). When it comes to technology readiness, the picture is far more encouraging, with availability of latest technologies assuming 39th place and technology adoption at 38th place in the ranking index (World Economic Forum 2012:325). Another favourable situation is the country's innovation ranking of 42 out of 144 countries (World Economic Forum 2012:325).

Seen in the context of the convergence of social and healthcare systems it needs to be recognised that a linkage exists between education in healthcare and the quality of the education system itself. Of similar pertinence is the linkage between innovation and technology application in support of healthcare service delivery. Innovative application of technology systems can play a fundamental role in enabling enhanced healthcare service delivery. These aspects will be dealt with in greater detail in analysing the systems in question. Of pertinence in this discussion is the realisation that a healthy workforce is vital to South Africa's ability to compete effectively in the global marketplace. This brings into question the importance attributed to $\mathrm{PHC}$ or a preventative stance as envisaged in the NHI policy document (DoH 2011a:5). The PHC philosophy refers to provision of a comprehensive service that includes preventative, promotive, curative and rehabilitative care (Western Cape DoH 2011:12). This is deemed important as good health affects social development and economic productivity through increased output of a healthy workforce, a longer 'work life' and an increase in labour force activity (DoH 2011a:19). Non-communicable diseases (NCD) account for $63 \%$ of all deaths worldwide and pose a substantial burden on both the healthcare system and the economy of the countries concerned. As a consequence, they also need to be factored into consideration from a healthcare system perspective (World Economic Forum 2011:6). They stem from a combination of modifiable and non-modifiable risk factors, the former including poor diet and physical inactivity, the use of tobacco products and alcohol - notably all factors that, to an extent, can be addressed within a PHCorientated context.

Assuming a preventative stance in addition to dealing with the curative aspect of healthcare management necessitates a more proactive PHC service-delivery system. This is where clinics, with their community-outreach focus, assume relevance in the proposed NHI initiative. The Western Cape $\mathrm{DoH}$ (2011:11) terms this to be a shift from focusing on the management of disease to the promotion of wellness. It is further accentuated that a central tenet of the PHC approach is the community's involvement in healthcare (Western Cape DoH 2011:11). Clinics, in effect, constitute the first level of contact of individuals, the family and community with the health system, bringing healthcare as close as possible to where people live and work (Australian Department of Health and Aging n.d.). It is here that mobile technologies have a particularly pertinent role to play in capturing patient demographic and health-specific information, according to respondents interviewed at a healthcare clinic. Healthcare workers make use of smartphones with appropriate applications loaded in order to capture and transmit the information acquired to the clinic's repository. The clinic physician interviewed during the course of this research study indicated that healthcare workers each have 200 households assigned to them and, as part of their community-outreach programme, they conduct home visits, during which individual family members' healthcare status and needs are determined and recorded on mobile devices. Where it becomes relevant that specific conditions requiring further tests or treatment exist, the people concerned are referred to their nearest clinic.

Healthcare workers are not only engaged in the monitoring and recording of a patient's healthcare status, but also play a very significant role in transferring healthcare information and knowledge to the community where they are engaged actively in the rendering of PHC services. In some instances the healthcare workers, where relevant, also make use of social workers and other similar professionally-qualified people, forming part of the community-outreach programme. An important point emphasised by a physician who was interviewed was that these healthcare workers stayed within these communities and were well aware of the conditions prevailing within their community setting. A distinctive advantage, therefore, is that the healthcare workers were ideally positioned to be able to interact with the people concerned, from a culture and language perspective.

The preceding brief description of the community-based PHC framework that is starting to unfold within the context of the specific clinic that formed the focus of the research study, tends to reflect the intention stated in the DoH's (2011a:23) NHI policy document, namely that in South Africa, PHC services will be re-engineered to focus on mainly community outreach services'. It is also articulated clearly that the composition of a defined comprehensive primary care package of services extends beyond the services traditionally provided in health facilities, such as clinics, community health centres and district hospitals (DoH 2011a:23). A clinic-centred community outreach and homebased PHC service system needs to be seen in the context of the larger healthcare systems framework presented in Figure 1. The technology systems will, of necessity, assume a more mobile orientation, with an accent on information systems and telemedicine applications. With this in mind, it needs to be noted that Mars and Seebregts (n.d.:4) attest to the reality of mHealth (mobile health) systems - with an increasing emphasis on medical informatics, telemedicine, surveillance and healthcare education - playing a very fundamental role in the transformation of a future healthcare dispensation. In so doing they do, however, caution that whilst $90 \%$ of the country is covered by mobile telephony, broadband penetration is low and bandwidth is expensive (Mars \& Seebregts n.d.:4). 
Clinics are linked to district hospitals and, from a technology perspective, the transfer of information between the two needs to be considered. The DoH (2010:18) envisions the strengthening of the District Health Information system (DHIS) and the progressive design and implementation of an appropriate electronic health record (EHR) system as being pertinent in this regard. Historically, patient medical records consisted of handwritten notes, typed reports, and test results stored in a paper-based filing system (Gartree 2011:14). A separate file was created and stored at each healthcare facility and radiological records were typically stored separately (Gartree 2011:14). A vast number of paper-based patient records have consequently come into being and South African hospitals are no exception in this regard. Alongside regulatory requirements for records retention (note the legal systems in Figure 1), hospitals are faced with moving forward with electronic charts whilst at the same time needing to manage the information contained in paper charts gathered during the transition so that they can be made available to caregivers (Hanover 2011:2). This challenge, as is emphasised by Hanover (2011:2), creates the need for hospitals and practices moving to the EHR to consider and adopt a records 'transition strategy' that allows them to access historical data whilst moving forward with their EHR. Often at a district hospital level, such a strategy has yet to be formulated and, as is so well-articulated by the Minister of Health, Dr Motsoaledi (DoH 2012):

[ $h$ ]istorically, health information systems in South Africa have been characterised by fragmentation and lack of coordination, prevalence of manual systems and lack of automation, and where automation existed, there was a lack of interoperability between different systems. Considerable resources were also invested in these systems that, in the final analysis, did not generate the expected returns on investment. (p. 5)

As a consequence, an outcome of the National Health Council meeting in August 2009 was the resolution that the acquisition of software solutions, which were not interoperable, should be halted until the eHealth strategy was finalised (DoH 2012:5). Within the private healthcare sector, EMR systems that facilitate the rendering of claims between healthcare service providers and medical aids have become a reality within what could best be described as being a broader national public sector EHR system vacuum. Research conducted by Mars and Seebregts (n.d.:4) reveals that only one-third of South Africa's provincial hospitals have some form of EMR system and that interoperability remains problematic. Recently, an enterprise content management (ECM) system was rolled out successfully at the Khayelitsha District Hospital in the Western Cape and a similar system is currently being deployed across the whole of the Tygerberg Academic Hospital in Bellville, one of South Africa's largest public-sector hospitals, located in the Western Cape (Datacentrix 2012:8). This, by implication, will mean that patient medical records can be transferred electronically from clinics supported by the Khayelitsha District Hospital, to the hospital itself, within the hospital and, where relevant, to the academic hospital involved in cases where clinical assistance was required or patients needed to be referred for specialist treatment. The DoH (2011b:9) acknowledges that in many public sector healthcare facilities, successful projects and initiatives are already showing results. Concern is, however, expressed that these islands of success are not currently integrated within a broader national framework.

\section{Weaving the healthcare service delivery systems, people and technology threads into a proposed healthcare fabric}

Although large sums of money have been used to procure health information, communication and technology (ICT) and health information systems (HIS) in South Africa in the past, the ICT and HIS within the public health system is not meeting the requirements to support the business processes of the health system thus rendering the healthcare system incapable of adequately producing data and information for proactive management and for monitoring and evaluating the performance of the national health system. (DoH 2010:10)

The above quotation, in a sense, attests to the preceding reality of islands of success that are not integrated within a broader national framework, yet envisaged in the DoH's NHI policy document (2011a:43) is the notion of an 'integrated and enhanced National Health Information System' based on an electronic platform. The current landscape, it is confirmed in the eHealth strategy document, is one of diverse proprietary systems in use within the public healthcare sector. With little integration between them and the private sector, however, these systems face the problem of system fragmentation (Chetty 2012; DoH 2012:14). The Western Cape Province appears progressively to be implementing a central hospital information system with a unique patient ID system, a pharmacy system, digital radiology and community healthcare clinics that access the same provincial-level patient identification (DoH 2012:14). However, seen in the context of a larger national information system it is unclear how these disparate systems will be integrated to support healthcare service delivery seamlessly across provincial and public-private sector boundaries. In terms of the privatesector health information systems, it is important to note that the focus still tends to be on billing and reimbursement and there is currently no formal integrated system to support patient care (DoH 2012:19). The need for national standards to achieve interoperability of healthcare information systems is raised by Chetty (2012). As alluded to previously, most patient-related information is still stored most frequently in paper format, which is essentially unstructured. What is ideally required are structured systems that facilitate patientspecific information retrieval and processing seamlessly across healthcare institutional boundaries, with due cognition of the need for the maintenance of security and confidentiality of patient information.

One of the principles encapsulated within the DoH's 1997 white paper for the transformation of the health system is that the National Health Information System (NHIS) should be coordinated nationally in order to support the effective delivery of services at all levels of the health system. It is further stressed that 'minimum standards for 
technology, coding systems, application software, the database management system, etc. will be adopted in the course of developing the NHISSA to ensure its user-friendliness, and facilitate the collation, analysis and use of data' (DoH 1997). An important point stemming from the preceding discourse is that merely plugging new, innovative, state-of-the-art healthcare information technology systems into an existing healthcare service-delivery framework will be fraught with difficulty and the risk of failure could be quite significant. Changes in the technology support systems need to take into consideration the impact thereof on the rest of the system framework depicted in Figure 1. The establishment of the DHIS would seem to support this contention, in that in concept its focus is broader than that of technology systems per se: it includes people, policies and procedures associated inherently with the healthcare systems strand of the healthcare systems framework (DoH 2011c:6).

Notably, one of the key challenges identified in the document relates to a lack of governance and standardisation of the DHIS, evidenced by ad hoc and uncoordinated implementation of new datasets. Another challenge highlighted is that of inadequate ICT infrastructure development that makes a shift to the use of web-based systems and remote information storage difficult (DoH 2011c:12). Of particular pertinence, however, is the noted shortage of people with the skills, experience and knowledge required (DoH 2011c:12). In analysing the concept of 'technology', Burgelman, Christensen and Wheelwright (2008:2) refer explicitly to the people aspect of knowledge, skills and artefacts as a factor that needs to be brought into consideration. Research conducted by Mars (2012:32) tends to confirm that within South Africa there are few practitioners trained in the effective utilisation of eHealth systems, a reality that will act as a constraint in weaving the technology thread into the healthcare systems fabric. Nowinski et al.'s claim (2007:S174) that the ultimate success of technology application emerges from a complex multidimensional interaction between the technology and its individual users, has definite relevance as well. The concept of 'users' within a healthcare dispensation is quite wide ranging and could well include not only healthcare workers and practitioners, but patients as well. The application of mobile technology within PHC settings, for instance, brings diverse technologies and, consequently, the associated skills required for their application, into consideration.

Research conducted by Zandieh et al. (2008:758) found that it needs to be recognised that healthcare practitioners differ significantly in terms of their capabilities relating to the use of electronic technological systems and a concern expressed by the researchers therefore relates to identifying the diverse technology support required by the people concerned. All of the respondents interviewed by Zandieh et al. (2008:758), however, were of the view that improving an individual's comfort level with the utilisation of information technology and decreasing resistance to changing workflow patterns constituted a very real challenge that needed to be addressed. A physician interviewed in the course of this research study confirmed that the training of nursing staff at the clinic in the use of the EMR system did not on its own offer a complete solution. In some instances they required additional backup support for dealing with technology issues that emerged whilst using the system in practice. An ICT practitioner involved in setting up and implementing the Enterprise Content Management change-management process at a district hospital indicated that an ICT call centre needed to be established in order to provide the healthcare practitioners with the support they required in dealing with technology issues that occurred during the day-to-day operations at the hospital. This finding, both from the literature and practice, lends support to the contention that the people strand that needs to be interwoven with the technology strand into the fabric of a new healthcare dispensation cannot just be ignored. Despite the apparent complexity and human changemanagement challenges associated with its implementation and utilisation, it is stressed by Gelmon and Droppers (2008:6) that information technology still offers the 'best hope for the future of healthcare'. It is similarly claimed by David and Jahnke (2004:73) that despite the human complexities involved, the appropriate deployment of technology will contribute significantly to a substantial improvement in the quality of healthcare delivered, the containment of cost and increased access to the services offered by the healthcare system.

An important element of the people thread that needs to be woven into the healthcare dispensation is that of fundamental realignment of the institution's culture, often described as 'the way we do things around here' (Du Plessis 2010:43; Hughes et al. 2012:360). The very notion of the adoption of a PHC approach and the introduction of mobile support technology systems implies a fundamental change in traditional practice or the ways things were traditionally done and consequently implies the need for a realignment of the healthcare cultural dispensation. Wise (2006) views the healthcare culture as being 'a whole way of life' and suggests that 'technology is not something separable from everyday life' and is therefore 'not separable from culture'. The implementation of the new NHI initiative in itself, with its multiplicity of interactive systemic changes, denotes a fundamental change in the life world of healthcare practitioners and, consequently, the cultures of the institutions involved. It is, for instance, claimed by David and Jahnke (2004:73) that the appropriate deployment of technology will contribute to a substantial improvement in the quality of healthcare delivered, the containment of cost and an increased access to the services offered by the healthcare system. Not, however, pertinently addressed in the DoH's policy document (2011a) are the organisational culture implications involved in the design, implementation, use and management of the new technologies in order to support healthcare service delivery. It could be argued, on the basis of the healthcare interactive systems framework, presented in Figure 1, that this is a seriously flawed omission.

Buchan (2011:321), in researching informatics for healthcare systems, concludes that although the technology application is reasonably predictable, the most challenging part of developing health information systems is that of overcoming cultural barriers. According to Yelton (2005:1), there appears to 
be an increasing recognition amongst healthcare practitioners of the need for a realignment of the culture within their institutions, yet moving from recognition to reality, it is argued by the researcher, seems to be somewhat more elusive. The researcher goes on to argue that a transformation of the healthcare culture is not a programme with a completion date, nor does it represent a quick fix solution (Yelton 2005:1). Rather, it is claimed by Yelton (2005:1), it needs to be seen as an 'on-going journey'. The challenge, the researcher suggests, is to 'institute cultural change that enables staff, clinicians and managers to feel empowered and adequately equipped' in order to bring about meaningful change in healthcare service delivery (Yelton 2005:3). Park and Kim (2009:22,33) similarly conclude that a culture that emphasises, flexibility, teamwork and cooperation can be more effective than a hierarchical culture that stresses work process and rules. Lluch (2011:852), however, contends that healthcare institutions tend to reflect a traditional hierarchical cultural orientation in practice.

A study of eight United States multispecialty medical groups by Nembhard et al. $(2012: 200,211)$ revealed a multiplicity and diversity of cultures within the groups concerned, but with a strong orientation toward rational and patientcentred values. It may be postulated that such diversity could be expected in practice, as the attributes giving rise to the cultural manifest will have evolved from the shared learning experience and interaction taking place between the healthcare practitioners concerned. This has particular relevance in terms of the broader community involvement encountered in a PHC dispensation as healthcare workers, social workers and related professional role players become part of a community with an emergent culture. In this sense, the culture transformation process articulated is one that reflects a complex adaptive system orientation, as opposed to the more traditional intentional reengineering approach that is encountered frequently in the literature. Far from the planned, ordered and well-managed view of traditional cultural transformation, Kimball (2005:11) describes the process as being fluid, unpredictable and filled with surprises.

Seen in the context of a PHC dispensation is the need to take cognition of the social interaction dynamics taking place between the various role players in clinic healthcare settings and their community outreach programs, as new values, beliefs and associated cultural attributes will emerge on an ongoing basis, as was alluded to previously by Yelton (2005:1). The PHC dispensation launched within the Pretoria Daspoort Clinic specifically brought a number of disciplines together in the community-outreach programme: social workers, medical students, healthcare workers, dental practitioners, engineers, ICT technologists and academics, all with well-established and diverse value systems. As noted by one of the physicians interviewed at the clinic, there emanated a unique strength from this diversity in terms of their having a common objective stemming from the $\mathrm{PHC}$ orientation in bringing services to people previously marginalised as a result of financial constraints To paraphrase Bennet and Bennet (2004:151) in this regard, the emergence is not random but is rather the result of interactions that settle down to internal coherence and patterns. It is a reality that gives meaning to Lessem and Schieffer's (2009) contention that:

$[w]$ hen we understand culture as the meaning giving system of a society then it becomes a current ever evolving force which can best be experienced in active engagement with today's human beings as well as with local nature, not with yesterday's historical beings. (p. 118)

Emerging from the discussion is the realisation that the NHI initiative, if seen from a broader systems-interaction perspective, entails far more than financial considerations; it embodies eHealth technologies and human systemic elements or threads that, when woven together, provide a sense of resilience to the emerging healthcare dispensation. An important aspect that has been highlighted is that of 'emergence' and, consequently, the need for a complex adaptive systems approach in managing the transformation to a new PHC-centred dispensation envisioned in the NHI policy document (DoH 2011a:5).

\section{The legal, financial and political threads of the National Health Insurance dispensation}

Given the strong legal architecture in the country, it is important that existing laws and, in particular, the guidance provided by the Constitution, are harnessed towards full and effective implementation of a NHI policy that advances access to quality health services. (Hassim 2010:205)

At the very core of the NHI initiative is a supportive legal, institutional and fiscal environment, the former accentuated clearly in Hassim's introductory quotation (2010:205). In effect, the legislative and financial support for the NHI initiative is determined politically. As accentuated by Goldberg (2012), 'the development of a national health system has been a focal point of discussions and proposals surrounding health sector reform, and is guided by the tenets of the African National Congress's (ANC) 1994 National Health Plan'. The NHI funding stems in fact from the annual budget submitted to and approved by parliament and, in view of the extensive funding requirements required, it will significantly feature in the 2013 budget to be presented to parliament by the Minister of Finance. Healthcare legislation emanates from the political process that has its genesis in the parliamentary process.

The importance attributed to the Constitution stems from the fact that it imposes an obligation on the State to provide access to healthcare services for all South Africans (Hassim 2010:205). Section 27 of the Bill of Rights in the Constitution (Republic of South Africa 1996:1255), for instance, stipulates that everyone has a right to healthcare and that the State must take reasonable legislative and other measures within its available resources to achieve the realisation of these rights (DoH 2011a:16). The range of legislation with a healthcare connotation is quite extensive. Hassim (2010:206), in this regard, states that there are well over 100 statutes that are administered by the DoH. These range from framework 
legislation, such as the National Health Act No 61 of 2003 (Republic of South Africa 2004), to statutes that govern health professionals and workplaces, to the regulation of medical schemes (Hassim 2010:206). It is an intricate and complex web of legislation with far-ranging implications for the existing and future healthcare dispensation. A case in point is the new healthcare workers that will form an essential enabling component of the PHC system and its community-outreach programme. Clarity as to the registration of such healthcare workers and their roles and responsibilities will need to be considered from a legislative perspective. The diverse webs of legislation weaving their way through the various systems or threads of the healthcare dispensation need to be reviewed and, where deemed necessary, adapted to both support and direct the establishment of the dispensation. It is a process that cannot be undertaken without reference to the envisioned future dispensation itself, which is reflected in the health policy document of 1997 (DoH 1997).

Gray, Vawda and Jack (2011:1) maintain that 'fundamental health policy document' remains the 1997 White Paper on the Transformation of the Health System in South Africa (DoH 1997). It informed the development of the NHI policy document, also known as the 'Green paper', and, according to Gray et al. (2011:3), no real effort has been made to update this document. The researchers, however, claim that there has been some progress made toward the implementation of the National Health Act (Act 61 of 2003) (Republic of South Africa 2004), which has been written in enabling terminology, necessitating substantial secondary legislation in the form of regulations to be made by the minister in order for it to be brought into operation. The health policy as encapsulated within the 'Green Paper' and its objectives (DoH 2011a:18) therefore currently serve as a key source of reference and direction in implementing a host of healthcare initiatives. Transitioning from an existing divided public- and private-healthcare dispensation to an integrated healthcare system requires a well-articulated implementation plan and, according to Gray et al. (2011:5), such a plan has not yet been articulated clearly by the DoH. It is argued within the context of this paper that such a plan would need to integrate the diverse systems reflected in Figure 1 into a holistic process that will enable the emergence of a future healthcare infrastructure that will meet the envisaged NHI objectives. Without the legislative enactment that will direct the process and provide the requisite funding, very little progress will therefore be made. In a sense, the implementation phases and their key features included in the Green Paper (DoH 2011a:48-51) serve to inform the process. Information management and systems support, as well as ICT infrastructure design and implementation, are reflected as elements that need to be addressed in the first phases of the project.

The funding of the host of activities to be undertaken in order to bring a new healthcare dispensation into effect brings the political domain into the spotlight, as it seems that some form of revised tax dispensation will need to be implemented. Pieterse (2009:34) attests to the sensitivity of these proposed changes and suggests that South Africans need to become engaged more actively in the NHI financing debate, as it will have an impact on all South Africans. The impact on medical aid schemes and the private healthcare sector also needs to be brought into consideration in this debate (Pieterse 2009:34). The lack of specialists, doctors and technologists also brings financial considerations into focus in terms of bring private sector resources into the public healthcare sector (Pieterse 2009:34). Bringing public healthcare services in line with those provided within the private sector will require a significant realignment of financial and human resources, necessitating some form of legal intervention. With this in mind, it is important to remember that certain constitutional concerns remain, especially those relating to the rights currently enjoyed by every South African, such as the right to freedom of association and administrative justice (Kirby 2011:32).

A detailed analysis of all the legislative and political elements concerned is not the objective of this paper. The accent here is to but to gain an insight into the importance of taking political, legal and financial system considerations into account in weaving the systems together that will eventually bring a new healthcare dispensation into being. The introduction of major public healthcare policies and shifts in fundamental socioeconomic systems will need to be consistent with the Constitution (Kirby 2011:32). It is maintained by Kirby (2011:32) that South Africans are not really opposed in principle to universal coverage in respect of healthcare services. However, it is stressed by Kirby (2011:32) that 'the enforcement of one single policy, driven by exclusionary rhetoric absenting proper debate is of concern. This is especially so in relation to the facts that accessing healthcare services is an emotional issue'. Interwoven people, legal, sociopolitical and financial systems feature quite pertinently in the preceding discussion and tend to lend support for the use of the healthcare systems framework reflected in Figure 1.

\section{Conclusion}

The brief analysis of the interdependencies and interaction that exist between the systems reflected in Figure 1 reveals that they need to be considered collectively in the design, implementation and management of the transition to a future healthcare dispensation. The insights gained from the restricted narrative enquiry tend to support that gained from the literature study in this regard. It is concluded that the healthcare systems management framework can serve as a source of reference for analysing the intersystem causeeffect relationships that will assume relevance in managing the transition process. It is also suggested that, in view of the numerous interactions and interdependencies that exist, it is nearly impossible to accurately foresee and predict the emergent outcomes, which would consequently imply the need for a complex adaptive systems approach. The importance attributed to the transition to a future healthcare dispensation is reflected in the South African government's National Development Plan, where it is stated that a definite milestone is the provision of 'affordable access to quality health care while promoting health and wellbeing'. An 
associated critical action identified in the plan is the phasingin of the NHI, 'with a focus on upgrading public health facilities, producing more health professionals and reducing the relative cost of private health care' (National Planning Commission 2012:24). The insights gained in this paper need to be seen in the context of this reality.

\section{Acknowledgments Competing interests}

The author declares that he has no financial or personal relationship(s) which may have inappropriately influenced him in writing this article.

\section{References}

Australian Department of Health and Aging, n.d., 'Primary health care reform in Australia - Report to support Australia's first national primary health care strategy', viewed 29 January 2013, from http://www.yourhealth.gov.au/internet/yourhealth/ publishing.nsf/Content/nphc-draftreportsupp-toc nphc-draftreportsuppch3 nphc-draftreportsupp-ch3-def

Bennet, A. \& Bennet, D., 2004, Organizational survival in the new world: The intelligent complex adaptive system, $\mathrm{KMCl}$ Press, Amsterdam.

Buchan, I., 2011, 'Informatics for healthcare systems', in K. Walsh \& J. Smith (eds.) Healthcare management, 2nd edn., pp. 321-336, McGraw-Hill, Maidenhead.

Burgelman, R.A., Christensen, C.M. \& Wheelwright, S.C., 2008, Strategic management of technology and innovation, International, 5th edn., McGraw-Hill, Boston.

Chetty, M., 2012, 'Information and communications technology in support of NHI. CSIR 4th Biennial Conference', viewed 15 February 2013, from http://researchspace. csir.co.za/dspace/bitstream/10204/6171/1/Chetty_2012.pdf

Datacentrix, 2012, 'Khayelitsha hospital creates and maintains electronic patient records', Infocentrix 2012(1):8-9, viewed 17 January 2013, from http://www.datacentrix. co.za/

David, Y. \& Jahnke, E.G., 2004, 'Planning hospital medical technology management', IEEE Engineering in Medicine and Biology Magazine, viewed 16 July 2013, from http:// ieeexplore.iee.org/xpl/login.jsp?tp=\&arnumber=1317985\&url=http\%3A\%2F\%2 ieeexplore.iee. org/xpl/login.jsp?tp=\&arnumber=1317985\&url=http $\% 3 A$
Fieeexplore.ieee.org\%2Fxpls\%2Fabs_all.jsp\%3Farnumber\%3D1317985

Department of Health, 1997, 'White Paper for the transformation of the health system in South Africa', viewed 15 February 2013, from http://www.doh.gov.za/show. php?id=3189

Department of Health, 2010, Negotiated service delivery agreement: 2010-2014 Department of Health, Pretoria, South Africa.

Department of Health, 2011a, 'National health Insurance in South Africa: Policy paper', viewed 12 December 2012, from https://www.pps.co.za/portal/Ditaba/NHI\%2O green\%20paper.pdf

Department of Health, 2011b, Fast track to quality: The six most critical areas for patient-centred care, Department of Health, Pretoria, South Africa.

Department of Health, 2011c, District health management information system (DHMIS) policy, Department of Health, Pretoria, South Africa.

Department of Health, 2012, eHealth strategy South Africa: 2012-2016, Department of Health, Pretoria, South Africa.

Du Plessis, J.W., 2010, 'Servitization: Developing a business model to translate corporate strategy into strategic projects', Unpublished research report, Graduate School of Technology Management, University of Pretoria.

Feachem, R.G.A., 2000, 'Health systems: More evidence, more debate', Bulletin of the World Health Organization 78(6), 715. PMid:10916908 PMCid:2560781

Gartree, R., 2011, Electronic health records: Understanding and using computerized medical records, 2nd edn., Pearson, Boston.

Gelmon, S.B. \& Droppers, S.O., 2008, 'Community health centers and electronic health records: Issues, challenges and opportunities', viewed 04 November 2012, from http://nwhf.org/images/files/Electronic_Medical_Record_Handbook.pdf

Gray, A., Vawda, Y. \& Jack, C., 2011, 'Health policy and legislation. South African Health Review', viewed 27 February 2013, from http://www.hst.org.za/sites/default/ files/Chap\%201\%20Health\%20Policy\%20pgs\%201-16.pdf

Goldberg, D., 2012, 'Health financing and the National Health Insurance in South Africa: An overview', viewed 26 February 2013, from http://www.polity.org.za/ Africa: An overview, viewed 26 February 2013, from http://www.polity.org.za/ article/health-financing
Hanover, J., 2011, 'Moving from paper to electronic health records: Optimizing the transition', viewed 23 March 2013, from http://www.ironmountain.com/ /media/ Files/Iron\%20Mountain/Knowledge\%20Center/Reference\%20Library/White\%20 Paper/Sponsored/IDC/Moving\%20from\%20Paper\%20to\%20Electronic\%20 Health\%20Records\%20IDC\%20Whitepaper.pdf

Hassim, A., 2010, 'National Health Insurance: Legal and civil society considerations', South African Health Review 2010, 205-208.

Hughes, C.M., Donnelly, A., Moyes, S.M., Peri, K., Scahill, S., Chen, C., et al., 2012 "The way we do things around here": An international comparison of treatment culture in nursing homes', Journal of the American Medical Directors Association 13(4), 360-367. http://dx.doi.org/10.1016/j.jamda.2011.03.005, PMid:21514243

Kimball, B., 2005, 'Cultural transformation in health care: A white paper that describes the complex nature of organizational culture and its role in health care organizations', viewed 16 June 2013, from http://www.healthws.com/graphics/cultural.pdf

Kirby, N., 2011, 'Driving the NHIS by exclusionary rhetoric', Without Prejudice 11(10), 30-32.

Lessem, R. \& Schieffer, A., 2009, Transformation management: Towards the integral enterprise, Gower, Burlington.

Lluch, M., 2011, 'Healthcare professionals'organisational barriers to health information technologies - a literature review', International Journal of Medical Informatics technologies - a literature review', International Journal of Medical Informatics

Mars, M., 2012, 'Building the capacity to build capacity in e-health in sub-Saharan Africa: the KwaZulu-Natal experience', Telemedicine Journal and e-Health 18(1), 32-37. http://dx.doi.org/10.1089/tmj.2011.0146, PMid:22150714, PMCid:3270048

Mars, M. \& Seebregts, C., n.d., 'Country case study for e-health South Africa', viewed 17 December 2012, from http://ehealth-connection.org/files/resources/County\%20 Case\%20Study\%20for\%20eHealth\%20South\%20Africa.pdf

Murray, C.J.L. \& Frenk, J., 2000, 'A framework for assessing the performance of health systems', Bulletin of the World Health Organization 78(6), 717-731.

National Planning Commission, 2012, 'National Development Plan: 2030', viewed 27 December 2012, from http://npconline.co.za/MediaLib/Downloads/Downloads/ Executive\%20Summary-NDP\%202030\%20-\%200ur\%20future\%20-\%20make\%20 it\%20work.pdf

Nembhard, I.M., Singer, S.J., Shorttell, S.M., Rittenhouse, D. \& Casalino, L.P., 2012, 'The cultural complexity of medical groups', Healthcare Management Review 37(3), 200-213. http://dx.doi.org/10.1097/HMR.0b013e31822f54cd, PMid:21934512

Nowinski, C.J., Becker, S.M., Renolds, K.S., Beaumont, J.L., Caprini, C.A., Hahn, E.A Peres, A., et al., 2007, 'The impact of converting to an electronic health record on organizational culture and quality improvement', International Journal of Medical Informatics 76 (Suppl 1), S174-S183. http://dx.doi.org/10.1016/j. ijmedinf.2006.05.016, PMid:16843717

Park, J.S. \& Kim, T.H., 2009, 'Do types of organizational culture matter in nurse job satisfaction and turnover intention?', Leadership in Health Services 22(1), 20-38. http://dx.doi.org/10.1108/17511870910928001

Pieterse, Q., 2009, 'NHI: All the snakes and ladders', Without Prejudice 9(11), 34-35.

Republic of South Africa, 1996, 'Constitution of the Republic of South Africa No. 108 of 1996', viewed 05 February 2013, from http://www.info.gov.za/documents/ constitution/1996/a108-96.pdf

Republic of South Africa, 2004, National Health Act No. 61 of 2003, viewed 04 December 2012, from http://www.info.gov.za/view/DownloadFileAction?id=68039

Weeks, R.V. \& Benade, S.J., 2011, 'Service science: A servitization systems perspective', paper presented at ISEM international conference on Industrial Engineering, System Engineering and Engineering Management for Sustainable Global Development, Spier, Stellenbosch, South Africa, 21-23 September.

Western Cape Department of Health, 2011, '2020: The future of healthcare in the Western Cape', viewed 15 February 2013, from http://www.westerncape.gov.za/ other/2011/12/healthcare_2020_-_9_december_2020.pdf

Wise, J.M., 2006, 'Technological culture', paper presented at the Asia Cultural Forum, Gwangju, Korea, viewed 25 July 2012, from http://www.cct.go.kr/data/acf2006/ mobile/mobile keynote2 Macgregor.pdf

World Economic Forum, 2011, The global economic burden of non-communicable diseases, World Economic Forum, Geneva, Switzerland.

World Economic Forum, 2012, 'The global competitiveness report 2012-2013', viewed 06 March 2013, from http://www3.weforum.org/docs/WEF_GlobalCompetitiveness Report_2012-13.pdf

World Economic Forum, 2013, Sustainable health systems: Visions, strategies, critical uncertainties and scenarios, World Economic Forum, Geneva, Switzerland.

Yelton, A.M., 2005, 'Making the journey toward cultural change in healthcare', viewed 16 February 2013, from http://www.gehealthcare.com/euen/services/docs/ Making_the_Journey_Toward_Cultural_Change_in_Healthcare.pdf

Zandieh, S.O., Yoon-Flannery, K., Kuperman, G.J., Langsam, D.L., Hyman, D. \& Kaushal, R., 2008, 'Challenges to EHR implementation in electronic- versus paper-based office practices', Journal of General Internal Medicine 23(6), 755-761. http:// dx.doi.org/10.1007/s11606-008-0573-5, PMid:18369679, PMCid:2517887 